\title{
A simulation of the behaviour of propane bulks on a grid platform
}

\author{
A. Laganà \& A. Costantini \\ Department of Chemistry, University of Perugia, Italy
}

\begin{abstract}
The increasing interest for the Ozone hole problem has prompted the European Union to ban the use of CFCs and HCFCs before the year 2015 (Copenaghen amendments). For this reason, intense research is presently being carried out to find alternative refrigerant fluids. Propane is one of these fluids and in order to understand its properties we carried out molecular dynamics simulations. In this paper we examine the results of our simulations and the difficulties met when implementing in parallel the suite of codes used for the molecular dynamics studies.
\end{abstract}

Keywords: propane, frigorigen fluids, molecular dynamics, thermodynamic properties

\section{Introduction}

Due to the recently approved Copenhagen amendments, the use of CFCs and HCFCs will be definitively banned before the year 2015. Hydrocarbons (HC) are being considered as an appropriate alternative. In particular HCs are considered as a long-term alternative refrigerant with no impact on global environment and in particular on stratospheric ozone depletion and global warming. Propane (also called R-290) is among them. It is therefore of great interest to carry out accurate computational simulations of propane bulks in order to rationalize their macroscopic properties and to single out in an ab initio way the theoretical foundations of their behaviour. Unfortunately this requires an amount of computing resources which is not always available. At present, a technology able to offer prospective affordable solutions to such a problem is Grid computing [1]. For this reason we decided to start a computational investigation aimed at constructing in an a priori fashion the state diagram of propane [2] on the EGEE- 
Grid platform [3]. To this end the DL_POLY [4] parallel software package was used in which the OPLS/AMBER all-atoms potential [5] was implemented. The paper is organized as follows: in section 2 the theoretical and computational machinery is sketched; in section 3 some indications on the performance of the used grid platform are given; in section 4 the results of the calculations are illustrated; in section 5 some conclusions are drawn.

\section{The theoretical and computational machinery}

The simulation is based on a Molecular Dynamics (MD) approach. In MD approaches the time evolution of an appropriate ensemble of particles is computed in order to work out from the microscopic behaviour of the system an estimate of the macroscopic properties. As already mentioned, in our investigation MD calculations were carried out using the DL_POLY suite of codes developed by W. Smith at the Central Laboratory for High Performance Computing of Daresbury [4]. As also already mentioned, the other pillar of our theoretical and computational machinery is the adoption of the OPLS/AMBER force field [5]. The OPLS/AMBER force field represents the nonbonded interactions as a combination of Coulomb plus a Lennard-Jones pair of terms as follows:

$$
V_{O P L S}=\sum_{i}^{\text {mol.a }} \sum_{j}^{\text {mol.b }}\left[\frac{q_{i} q_{j} e^{2}}{r_{i j}}+4 \epsilon_{i j}\left(\frac{\sigma_{i j}^{12}}{r_{i j}^{12}}-\frac{\sigma_{i j}^{6}}{r_{i j}^{6}}\right)\right] f_{i j}
$$

where $q_{i}$ and $q_{j}$ are the charges of atom $i$ and $j$ respectively, $e$ is the charge of the electron, $\epsilon_{i j}, \sigma_{i j}$ and $r_{i j}$ are the depth, the equilibrium and the actual distance respectively between atom $i$ and $j$. In eq. ( $1 f_{i j}$ is always taken equal to 1 for all the $i j$ interactions. The same expression is used for intramolecular nonbonded interactions between all pairs of atoms separated by three or more bonds. The intermolecular interaction for angular (eq. (2)) and torsional (eq. (3)) motions is represented as

$$
V_{\text {angle }}=\sum_{\text {angle }} K_{\theta}\left(\theta-\theta_{e q}\right)^{2}
$$

with $\theta$, the bending angle, having an equilibrium, $\theta_{e q}$, value of $109.5^{\circ}$ and

$$
\begin{aligned}
& V_{\text {dihedral }}=\sum_{i} \frac{V_{1}^{i}}{2}\left[1+\cos \left(\phi_{i}\right)\right]+\frac{V_{2}^{i}}{2}\left[1+\cos \left(2 \phi_{i}\right)\right]+ \\
& +\frac{V_{3}^{i}}{2}\left[1+\cos \left(3 \phi_{i}\right)\right]
\end{aligned}
$$

with $\phi_{i}$, being the dihedral angle and $V_{1}, V_{2}$ and $V_{3}$ the coefficients of the Fourier series. In the present system all the bonds have been considered constant and the total energy is taken as

$$
V=V_{O P L S}+V_{\text {angle }}+V_{\text {dihedral }}
$$


The value of the parameters used in the adopted OPLS/AMBER potential is given in Tables 1 and 2 [5].

Table 1: Values of the parameters of the OPLS/AMBER bending and torsion potentials of the Propane system.

\begin{tabular}{ccc}
\hline \multicolumn{3}{c}{ Bending } \\
\hline Group & $\theta_{e q}$ & $K_{\theta}$ \\
& $/ \circ$ & $/{\text { kcal } \mathrm{mol}^{-1}}^{-}$ \\
\hline$H-C-H$ & 109.5 & 35.00 \\
$H-C-C$ & 109.5 & 35.00 \\
$C-C-C$ & 109.5 & 40.00 \\
\hline
\end{tabular}

\begin{tabular}{cccc}
\hline \multicolumn{3}{c}{ Torsion } & \\
\hline Group & $V_{1}$ & $V_{2}$ & $V_{3}$ \\
& $/$ kcal mol $^{-1}$ & $/$ kcal mol $^{-1}$ & $/$ kcal mol $^{-1}$ \\
\hline$H-C-C-H$ & 0.000 & 0.000 & 0.318 \\
$H-C-C-C$ & 0.000 & 0.000 & 0.366 \\
\hline
\end{tabular}

Table 2: Parameters for the OPLS/AMBER nonbonded potential of the Propane system.

\begin{tabular}{lccc}
\hline Atom & $\mathrm{q} / e^{-}$ & $\sigma / \dot{A}$ & $\epsilon /$ kcalmol $^{-1}$ \\
\hline$C, \mathrm{RCH}_{3}$ & -0.180 & 3.650 & 0.0635 \\
$C, \mathrm{R}_{2} \mathrm{CH}_{2}$ & -0.120 & 3.650 & 0.0635 \\
$\mathrm{H}$ & 0.060 & 2.585 & 0.0287 \\
\hline
\end{tabular}

\section{Performances of the used grid platform}

To evaluate the performance of the used platform, six different EGEE-Grid computer clusters were used. In order to evaluate the elapsed time of each simulation and the related speed-up for each cluster, we ran the calculations sequentially on one node and in parallel on 2 and 4 nodes. Measured elapsed times and speedups are plotted in Fig. 1 and 2 respectively. As shown by the figures the parallel performance of some clusters of the EGEE-Grid is very close to the 
ideal value due to their dedicated usage. Deviations from it occurring in the other clusters are mainly due to the time sharing regime adopted by them. This means that the parallel performances of each EGEE-Grid cluster strictly depend on the adopted regime. In order to evaluate more in detail the parallel performance of each cluster and the waiting time intercurring between the scheduling and the running of a process we restricted parallel calculations to two nodes. To carry out a statistical analysis we ran 50 parallel jobs (see Table 3). As apparent from the table, more than $70 \%$ of the jobs ran properly and only $26 \%$ were aborted. Abortion is due for $62 \%$ of the cases to communication errors between the nodes of the same cluster, for $23 \%$ to errors internal to DL_POLY occurred during the running and for $15 \%$ a wrong scheduling.

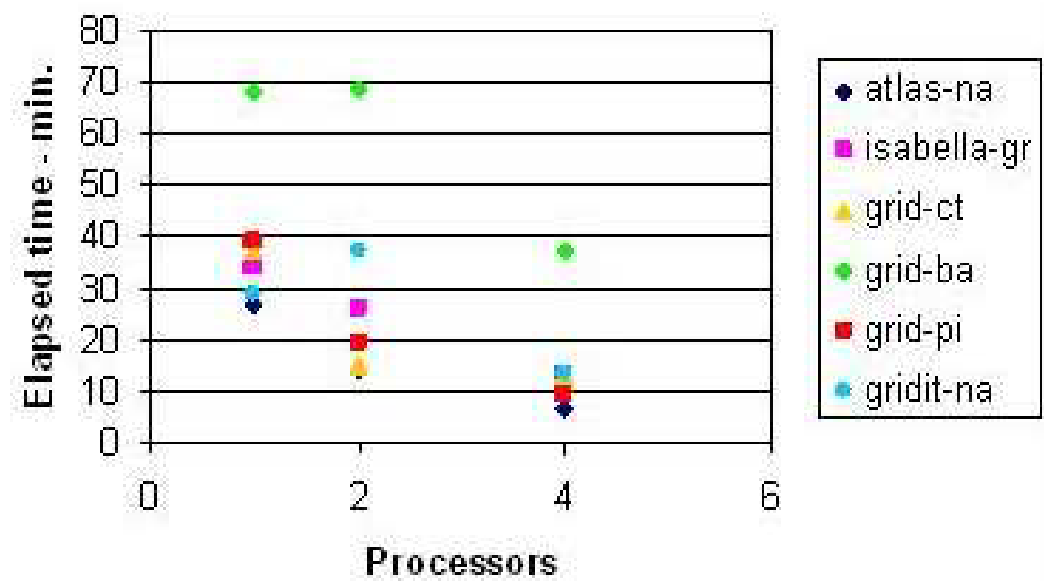

Figure 1: Elapsed time measured on six different EGEE-Grid clusters (listed in the right hand side of the graph) plotted as a function of the umber of processors used.

\section{Calculations and results}

All the calculations were carried out using the same $n p t$ statistical ensemble at two different pressures and temperatures in order to simulate the liquid phase at the equilibrium and out of equilibrium conditions for a bulk system of 288 molecules of propane. All the simulations were performed for a time duration of $1 \mathrm{~ns}$. As shown by Table 4, the calculated value of the density of the system gets closer to the experimental data given in literature $\left(582 \mathrm{Kg} \mathrm{m}^{-3}\right.$ at $\mathrm{T}=230 \mathrm{~K}$ at $\mathrm{P}=1.013$ bar [6]) when going from 10 bar to 1 bar. An important aspect to single out here is the fact that the pressure calculated at the end of the simulation does not agree with the imposed pressure (0.8 bar larger). From values reported in Table 4 it appears clearly that this difference $(\Delta P)$ is not constant. $\Delta P$ decreases, in fact, as 


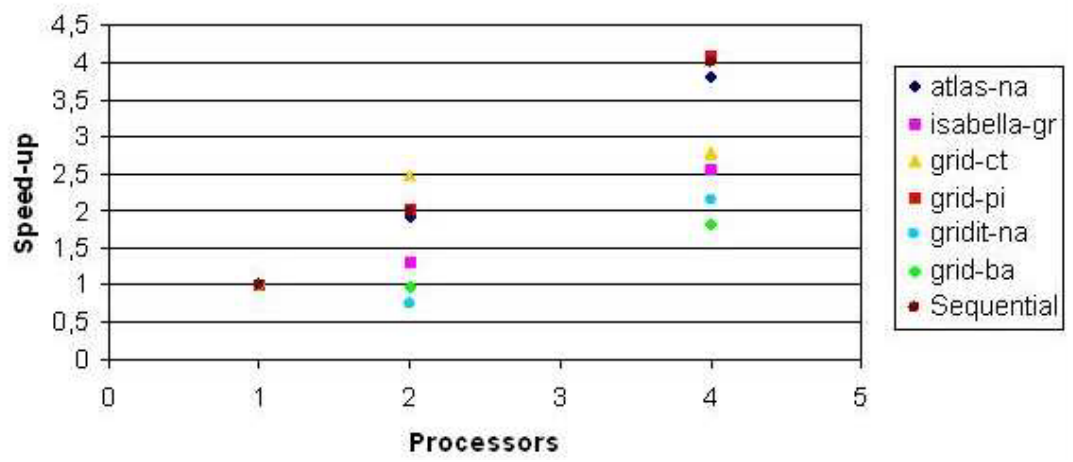

Figure 2: As in Figure 1 for speedups.

Table 3: Statistics on EGEE-Grid submissions out of 50 jobs.

\begin{tabular}{ccc}
\hline Job status & Number & $\%$ \\
\hline Success & 37 & 74 \\
Aborted & 13 & 26 \\
\hline \hline Cause for abort & Number & $\%$ \\
\hline Communic. error & 8 & 62 \\
DL_POLY error & 3 & 23 \\
Scheduler error & 2 & 15 \\
\hline
\end{tabular}

the pressure increases. Another set of calculations was carried out at $100 \mathrm{~K}$ at a different pressure in order to evaluate the property of the system out of equilibrium (Table 5). As shown by the Table, also the density of the system out of equilibrium decreases in going from 10 bar to 1 bar. However the effect of the pressure on the system seems to be stronger if the temperature decreases. Further calculations are being carried out to single out the microscopic reasons for such an effect.

\section{Conclusion}

We have studied the propane system in liquid phase using the OPLS/AMBER definition of the force field on the EGEE-Grid platform in order to reproduce the properties of the system and, at the same time, to evaluate the performance of the parallel calculations. We found that the calculated value of the density of the system gets closer to the data given in literature (when going from 10 bar to 1 bar) and we also found a substantial difference between the calculated average pressure and imposed pressure values since the former strongly dependent from the temperature and the density of the system. Further investigations suggested by our 
Table 4: Thermodynamic properties of Propane bulk system calculated after $1 \mathrm{~ns}$ of simulation time at $230 \mathrm{~K}$.

\begin{tabular}{ccc}
\hline Pressure / bar & Density / $\mathrm{Kg} \mathrm{m}^{-3}$ & $\Delta P /$ bar \\
\hline $1.0035 \pm 28$ & $584.2 \pm 4.4$ & 0.8705 \\
$1.9768 \pm 27$ & $585.2 \pm 5.6$ & 0.8568 \\
$3.9590 \pm 28$ & $585.4 \pm 5.3$ & 0.8394 \\
$7.9393 \pm 26$ & $585.2 \pm 5.4$ & 0.8196 \\
$9.9444 \pm 28$ & $585.7 \pm 6.6$ & 0.8243 \\
\hline
\end{tabular}

Table 5: Thermodynamics properties of Propane bulk system calculated after $1 \mathrm{~ns}$ of simulation time at $100 \mathrm{~K}$.

\begin{tabular}{ccc}
\hline Pressure / bar & Density / $\mathrm{Kg} \mathrm{m}^{-3}$ & $\Delta P /$ bar \\
\hline $1.0509 \pm 25$ & $722.9 \pm 4.7$ & 0.9179 \\
$2.0362 \pm 23$ & $723.9 \pm 5.3$ & 0.9162 \\
$4.0492 \pm 25$ & $724.2 \pm 5.6$ & 0.9292 \\
$8.0334 \pm 24$ & $724.5 \pm 5.2$ & 0.9134 \\
$10.047 \pm 26$ & $725.0 \pm 5.8$ & 0.9273 \\
\hline
\end{tabular}

results are those aimed at understanding whether this difference is due to the nature of the repulsive interaction adopted by the model potential. We found also high statistical errors which affect the pressure values. Other investigations suggested by our results are those concerned with the need for increasing of the simulation time in order to obtain a better average value of pressure, a better definition of the variation of $\Delta P$ using a more extended range of temperatures and pressure and a tuning of the force field of the system in order to exert a better control of the effect of the pressure on the system.

\section{Acknowledgements}

This work has been supported financially by MIUR, ASI, CNR and COST CMST (action D37 GRIDCHEM). Specific mention needs to be made to the Italian MIUR FIRB Grid.it project (RBNEOIKNFP) on High Performance Grid Platform and tools and to the MIUR CNR Strategic Project L 499/97-2000 on High Performance Distributed Enabling Platforms. 


\section{References}

[1] Storchi, L., Manuali, C., Gervasi, O., Vitillaro, G., Laganà, A., Tarantelli, F.: Lecture Notes in Computer Science 2658 (2003) 297-306

[2] Costantini, A., Laganà, A., Pirani, F.: Lecture Notes in Computer Science 3980 (2006) 738-743

[3] EGEE website: http://public.eu-egee.org

[4] Smith, W., Forester, T.R.: DL_POLY2: a general-purpose parallel molecular dynamics simulation package. J. Mol. Graph. 14 (3) (1996) 136-141

[5] Jorgensen, W. L., Maxwell, D.S., Tirado-Rives, J.: J. Am. Chem. Soc. 118 (1996) 11225-11236

[6] Air liquid group website: http://www.airliquide.com 